\title{
The Upgraded CDF Front End Electronics for Calorimetry
}

\author{
G. Drake, D. Frei, S. Hahn, C. Nelson, S. Segler and W. Stuermer
}

Fermi National Accelerator Laboratory

P.O. Box 500, Batavia, Illinois 60510

November 1991

* Presented at the IEEE Nuclear Science Symposium, Santa Fe, New Mexico, November 2-9, 1991. 


\section{Disclaimer}

This report was prepared as an account of work sponsored by an agency of the United States Government. Neither the United States Government nor any agency thereof, nor any of their employees, makes any warranty, express or implied, or assumes any legal liability or responsibility for the accuracy, completeness, or usefullness of any information, apparatus, product, or process disclosed, or represents that its use would not infringe privately owned rights. Reference herein to any specific commercial product, process, or service by trade name, trademark, manufacturer, or otherwise, does not necessarily constitute or imply its endorsement, recommendation, or favoring by the United States Government or any agency thereof. The views and opinions of authors expressed herein do not necessarily state or reflect those of the United States Government or any agency thereof. 


\title{
The Upgraded CDF Front End Electronics for Calorimetry
}

\author{
G. Drake, D. Frei*, S. R. Hahn, \\ C. A. Nelson, S. L. Segler, W. Stuermer \\ Fermi National Accelerator Laboratory, Batavia, IL ${ }^{* *}$
}

\begin{abstract}
The front end electronics used in the calorimetry of the $\mathrm{CDF}$ detector has been upgraded to meet system requirements for higher expected luminosity. A fast digitizer utilizing a 2 $\mu \mathrm{Sec}, 16$ bit $\mathrm{ADC}$ has been designed and built. Improvements to the front end trigger circuitry have been implemented, including the production of 900 new front end modules. Operational experience with the previous system is presented, with discussion of the problems and performance goals.
\end{abstract}

\section{INTRODUCTION}

The CDF detector has been described previously [1]. It consists of several different components, including tracking chambers, electromagnetic shower counters, hadron calorimeters, and muon chambers. The detector is divided into three regions. The central detector covers the region from $30^{\circ}$ to $150^{\circ}$, as measured from the beam direction. The plug detector forms the pole pieces for the solenoidal magnet, and covers the region $10^{\circ}$ to $30^{\circ}$. The forward detectors cover the forward and backward regions, from $2^{\circ}$ to $10^{\circ}$. An important design goal for the original data acquisition system was to unify the front end electronics as much as possible, despite the large number of detector systems. The result was the development of only two electronic subsystems, one for calorimetry and another for tracking.

During the last collider run in 1989, there were four levels to the CDF trigger system. Three of these levels were capable of creating "dead time," or missed beam crossings. The Level $0(\mathrm{~L})$ trigger signals the detection of a beam-beam interaction. The trigger detects the small-angle scattering coming from the interaction region. The L0 trigger does not produce dead time by itself, since the L0 decision is made within $100 \mathrm{nSec}$ after the beam crossing. If an interaction is detected, a LO Accept signal is sent to the Level 1 (L1) system [2]. The L1 trigger performs a coarse energy deposition measurement using fast signals called "fast outs" from the electronics associated with the calorimeters. If certain conditions are met, such as a single tower over a predefined threshold, then a L1 Accept is sent to the Level 2 (L2) system. The L1 Accept stops the data acquisition cycle, but does not initiate digitization or read out of the data. The L2 system makes a more precise measurement of energy deposition using the same fast out

\footnotetext{
* Now at University of Bern, Switzerland

** Operated by the Universities Research Association, Under

Contract with the U.S. Dept. of Energy
}

signals. If L2 conditions are met, then a L2 Accept is sent to the data acquisition system, which begins the digitization and read-out process. The digitized data is collected from all over the entire detector, and funnelled into the Event Builder [3] before it passes into the Level 3 (L3) system. This is the last stage of filtering before the event is written to tape for off-line analysis. Once the data is read out from the front end, the system is ready to acquire the next event. The L3 system creates dead time only when the L2 acceptance rate is higher than the rate at which $\mathrm{L} 3$ can make decisions.

The luminosity of the proton-antiproton collisions during the 1989 run was approximately $1 \times 10^{* *} 30 / \mathrm{cm}^{2} / \mathrm{sec}$. It is expected to increase to $5 \times 10^{* * 30} / \mathrm{cm}^{2} / \mathrm{sec}$ for the $1992 \mathrm{run}$. The interval between beam crossings will remain at $3.5 \mu \mathrm{Sec}$, but the average number of interactions per crossing is expected to increase from less than one to approximately 1.5 . The result is an increase in the rate at which data is acquired, imposing certain performance criteria on the front end electronics.

During the 1989 run, the dead time associated with reading out the front end was approximately $14 \mathrm{mSec}$ on the average. This corresponded to $7.8 \%$ of the total time necessary to process an event into the $\mathrm{L} 3$ trigger system. The L1 trigger decision required $7 \mu \mathrm{Sec}$. When a L0 Accept occurred, the system was dead during the very next crossing to allow the L1 trigger to make a decision. For the next run, with the luminosity increasing by a factor of 5 and interactions occurring every beam crossing, the present Level 1 trigger would cause the system miss half of the beam crossings. To improve the trigger performance and the overall system throughput, several upgrades have been designed and implemented in the front end electronics involved with calorimetry: new hardware to digitize and scan the calorimetry signals faster; new hardware to form Level 1 trigger signals faster; and new data acquisition code to optimize the hardware. The goals were to increase the performance while maintaining the precision of the 16 bit system, and to minimize the cost and perturbation to a working system.

\section{THE RABBIT SYSTEM}

The front end electronics used for the calorimetry in CDF is a customized system known as RABBIT [4-5], which consists of two parts. The first component consists of a RABBIT crate, which contains various front end instrumentation modules, a crate controller/digitizer called the EWE, and a timing module called the BAT. The crates are typically located less than four meters away from the detector components. There is one RABBIT crate on each of 48 
wedges in the central region of the detector, as well as an additional 32 used for the forward calorimetry and 32 on the endwalls and plugs. There are 17 additional crates in the system used for other types of instrumentation, giving a total of 129 RABBIT crates. The second component is the MX, a high-speed, programmable computer designed to control and read out the RABBIT crates. The MX computers are located in the counting room and are connected to the RABBIT crates by means of a 70 meter long cable called the UBUS. There is one MX for every two RABBIT crates.

The RABBIT system was designed to accommodate a wide variety of modules. It was anticipated that each detector would require a different type of instrumentation. This provided the opportunity to optimize the front end electronics for each detector to achieve the best possible performance in terms of gain, input impedance, frequency response, and trigger system interface. Despite attempts to minimize the number of different designs, almost every detector has had a different module produced for it. The extreme case is the electromagnetic and hadronic calorimeters for the plug and forward regions, for which 15 different variations of one module design were required.

The front end modules contain charge-sensitive amplifiers, time-to-voltage amplifiers, voltage and temperature monitors, and other types of instrumentation. Each channel contains track-and-hold circuitry, which is controlled by the BAT timing module residing in the crate. The BAT receives a timing signal called Clear \& Strobe (C\&S) from the system clock which is synchronized with the beam crossing. Each front end channel has two track-and-hold circuits; one for measuring the output voltage just before a beam crossing, the other for measuring the voltage after a fixed delay from the beam crossing, thereby setting the integration time. This double-correlated sampling technique results in a high degree of insensitivity to baseline drifting and low-frequency noise. See Fig. 1.

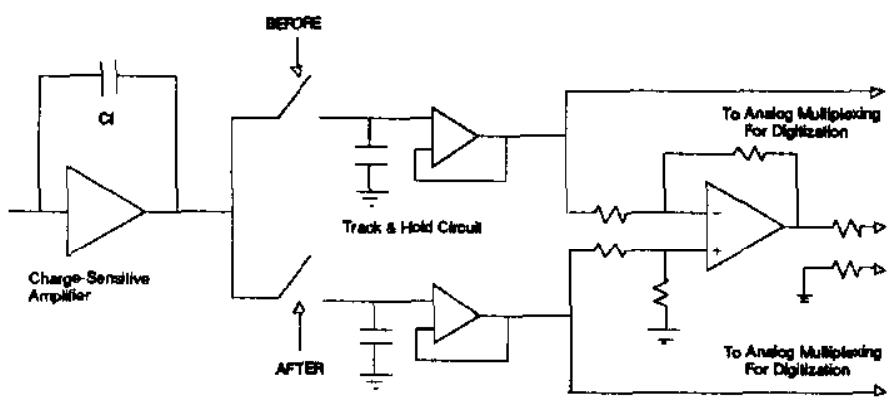

Fig. 1. RABBIT Front End Channel with Double-Correlated Sampling and Fast Out

Selected modules send fast analog signals called "fast outs" from the output of the track-and-hold circuitry to the Level 1 trigger system in the counting room, which is 70 meters away from the detector. The Level 1 trigger system constructs weighted analog sums over the entire detector to measure energy deposition and determine whether an interesting event has occurred. The fast out signals are summed over several towers and given $\sin \theta$ weighting so as to sum transverse energy deposition. If Level 1 conditions are satisfied, the sampling of the front end instrumentation is suspended, and the Level 2 trigger system is activated. It uses the same fast out signals in conjunction with information from the tracking system to further refine the measurement . If Level 2 conditions are met, a "Start Scan" message is generated, activating the data acquisition system.

The scan list for each crate is kept in the associated MX. When the data acquisition system receives a "Start Scan" message, each MX sends digital information for the first channel in the crate to be digitized down to the EWE crate controller. The EWE sends out the digital address across the RABBIT backplane. The selected module in the crate responds by addressing the selected channel and multiplexing the sampled output voltage in differential form out onto the backplane. The EWE receives the differential voltages, takes the difference, and then performs a 16 bit analog-to-digital conversion. The resulting digital word is driven out the EWE to the MX over the UBUS. The process is repeated until all channels in the crate have been digitized. (There are approximately 500 channels in each crate.) The system is designed so that all 130 RABBIT crates in the system digitize in parallel, albeit asynchronously. When all the MX computers are done, the system becomes live again, the trackand-hold circuitry is reset from the previous hold mode, and the system is ready for the next event.

\section{SYSTEM UPGRADES}

The old EWE II utilized a $17 \mu \mathrm{Sec}, 16$ bit linear ADC, which was among the fastest 16 bit converters available at the time (1983). An additional $3 \mu \mathrm{Sec}$ was provided to allow signals to settle. Because the ADC was too slow to digitize every channel, the digitizer module performed pedestal subtraction and sparsification before conversion using analog circuitry so that only those signals above threshold were digitized. The pedestal and threshold information was downloaded with the channel address from the MX on a channel by channel basis. The digital words for the pedestal and threshold were converted to analog voltages using digital-to-analog converters. The pedestal voltage was subtracted from the differential channel voltage, and the result presented to a comparator to compare with the threshold voltage. If the pedestal-subtracted channel voltage was greater than the threshold voltage, then the pedestal-subtracted channel voltage was digitized. Otherwise, the EWE sent a message back to the MX that it was done, but had no data. Thus, only those channels above threshold incurred the costly digitization time.

Because the crates are inaccessible during the run, the system was designed to minimize failure modes by minimizing the amount of intelligence and electronic complexity in the RABBIT crates. One decision was to defer any sequencing capability or use of a local scan list in the original EWE II, which operated on one channel at a time based on information sent from the MX. Another decision was to have only one ADC per crate. All channels in the crate are digitized by a single ADC in serial fashion. This philosophy preserved the measurement precision by reducing synchronous digital activity, made the digitizer board relatively 
simple, kept the per channel cost low, and reduced calibration problems.

The RABBIT system has been highly successful, having been operated through three physics running periods over the course of six years. However, several features of the design eventually became limiting factors in increasing the data rate. First, the scheme required a substantial amount of communication between the MX and the EWE, in which round trip cable delays contributed substantially to the scan time. The problem was exacerbated because the UBUS had been made bi-directional, using current-drive ECL drivers. When the data bus is switched from read to write, $800 \mathrm{nSec}$ settling time is needed for the data to become valid at the receiving end of the cable. Second, while the time spent digitizing during the scan was minimized by converting only those channels above threshold, the total time to read out the front end was determined by the crate with the highest occupancy for a given event. The forward detector regions, having the highest occupancy, took the longest to read out. Third, there was almost no overlapping or pipelining of activity. The EWE II was a slave, responding only to the current commands from the MX. The only overlap of activity was due to the ability of the MX to control and read out the two RABBIT crates connected to it in parallel.

To improve this performance, a new EWE, designated EWE III, was designed with emphasis on minimizing the perturbation to the overall system. The EWE III contains a high-speed 16 bit linear ADC, the Burr Brown $\mathrm{ADC} 701 / \mathrm{SHC} 702$, which digitizes in $2 \mu \mathrm{Sec}$ while maintaining \pm 1 lsb accuracy [6]. Because the digitization is 9 times faster, the philosophy of doing pedestal subtraction and over-threshold testing using analog techniques was abandoned. Combined with other hardware and software improvements, it is now faster to digitize every channel and do the pedestal subtraction and sparsification digitally in the MX.

Another improvement allows the EWE III to perform a limited amount of automatic sequencing at the cost of a small increase in complexity. While not every module location in every RABBIT crate is occupied, it is generally true that the channels to be digitized on a selected card are contiguous. A digital counter was implemented on the EWE III which operates in count down mode. The MX sends the address of the module to be digitized to the EWE over the UBUS as before. It also loads the subaddress of the last channel on the card into the counter, and issues the first convert command. The EWE digitizes the channel voltage, sends the digital result to the MX, and automatically decrements the counter. The $M X$ reads the $A D C$ word, and then issues the next convert command. There is still one round trip delay per channel on the UBUS, but the channel settling time overlaps with it. Also, the UBUS does not change direction with every channel. The direction is changed only when a new module is selected. This reduces by a factor of 30 the time spent waiting for the UBUS data to settle and become valid.

The data acquisition code has also been changed substantially to accommodate the new hardware. The MX stores the raw data in memory until all the channels in the crate have been digitized. It then sends a "Done" signal to the system before it performs the pedestal subtraction and overthreshold testing on the data. The system becomes live during this activity, allowing the front end to acquire the next event. While the system can become dead if the event rate is too high, this is another level of pipelining incorporated in to the system to help improve the data acquisition rate.

Another improvement to the MX code is that pedestals are now acquired in the same way as the data. Previously, a pedestal run was performed by digitizing all channels in a crate for a given event. After each pedestal event, the data was read out by the MX computers and processed through the system. The list was executed a minimum of 300 times to provide enough statistics to compute average values. There was a high degree of periodicity in this operation, making the pedestals sensitive to coherent effects such as UBUS direction changes and repetitious gating cycles. During the last physics run however, data sparsification occurred on the EWE II, and any coherent effects tended to be negated by the random nature of the data. This, combined with other subtle effects, produced small errors in the physics data that needed to be corrected offline. With the new EWE III and data acquisition code, the difference in time structure of the data acquisition no longer occurs since all channels are digitized in both types of runs.

Another area in which improvements have been made to the system concern the Level 1 trigger driver circuitry. One of the problems with the double-correlated track-and-hold scheme is that a pulse is produced on the output during the integration time. This occurs because charge is injected from the analog switches onto the holding capacitors when the switches open. During the integration time the circuit is unbalanced, creating the pulse. The pulse decays when the second switch opens at the end of the integration time, as shown in Fig. 2. This pulse must settle to the desired Level 1 trigger sensitivity of $500 \mathrm{MeV}$ within $1.9 \mu \mathrm{Sec}$ after the beam crossing on the receiving end of the cable, in order to provide time to make a Level 1 decision and not miss the next beam crossing if the event is rejected.

The PMADC modules used with the photomultipliers have been modified to achieve this settling specification. New op amps with $40 \mathrm{MHz}$ gain-bandwidth products have been substituted for the older, slower amplifiers. New pole-zero compensation was added to better match the amplifier to the cable.

The electronics used with the forward and plug calorimeters was completely redesigned, primarily to improve the trigger drivers. The new modules are called GPA, and make use of a current-feedback op amp, the AD844, to speed up the signal drive. In addition, compensation circuitry has been added to the track-and-hold circuits to reduce the effect of the charge injection from the analog switches. This is shown in Fig. 3. 


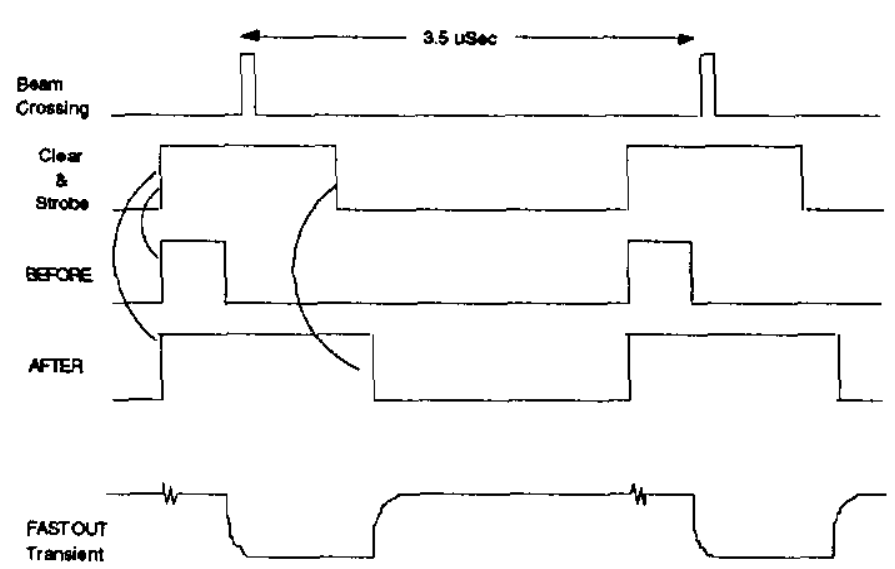

Fig. 2. Fast Out Response from Double-Correlated Sampling.

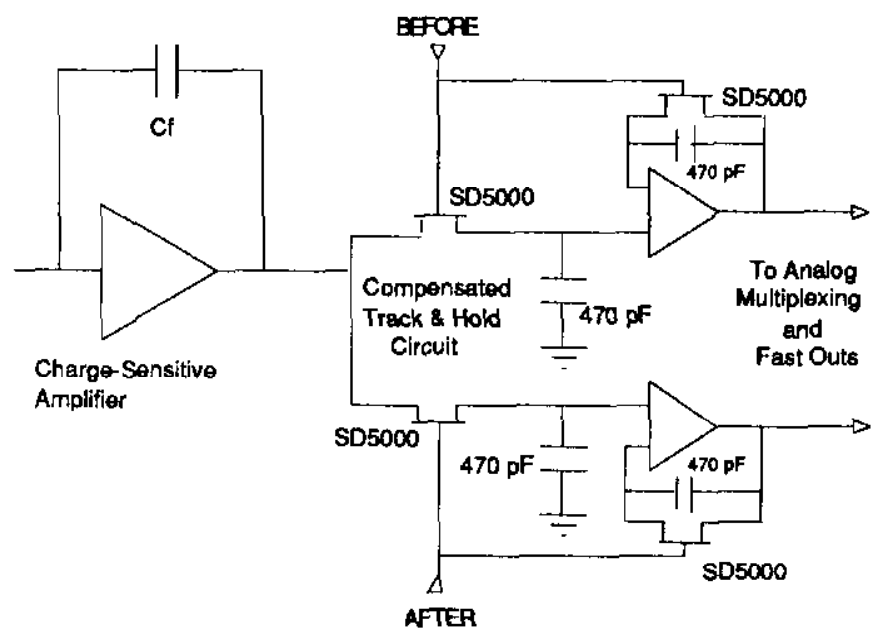

Fig. 3. Analog Switch Charge Injection Compensation used on the GPA Modules.

\section{TEST RESULTS}

Two hundred new EWE III modules have been produced. They have been checked out, calibrated, and installed in the system. The new data acquisition code that resides in the MX computers has been completed and debugged. Nine hundred new GPA modules have been produced as well, and are also checked out and installed. While the commissioning is still in progress, some preliminary system tests have been completed.

With the new hardware and software, all channels are now digitized for each event so that the scan times remain constant independent of occupancy. Using the real-time clock in the MX, the scan times have been measured, both before and after the improvements. Fig. 4 shows a histogram of the worstcase scan time for each event taken from a 10 hour running period during the last physics run. Note that this includes all of the MX computers in the system, and that a given MX may not always take the longest time to read out. While there is not yet any data to show how much time will be required to read out the upgraded system, it is useful to compare with the time necessary to read out pedestals, since the data acquisition process is now the same for both cases. The worst case scan time is now $1.99 \mathrm{mSec}$, corresponding to the $\mathrm{MX}$ with the largest number of channels associated with it. This is to be compared with the average worst case time of $14 \mathrm{mSec}$ before the upgrade, with the longest scan time measured at $18 \mathrm{mSec}$.

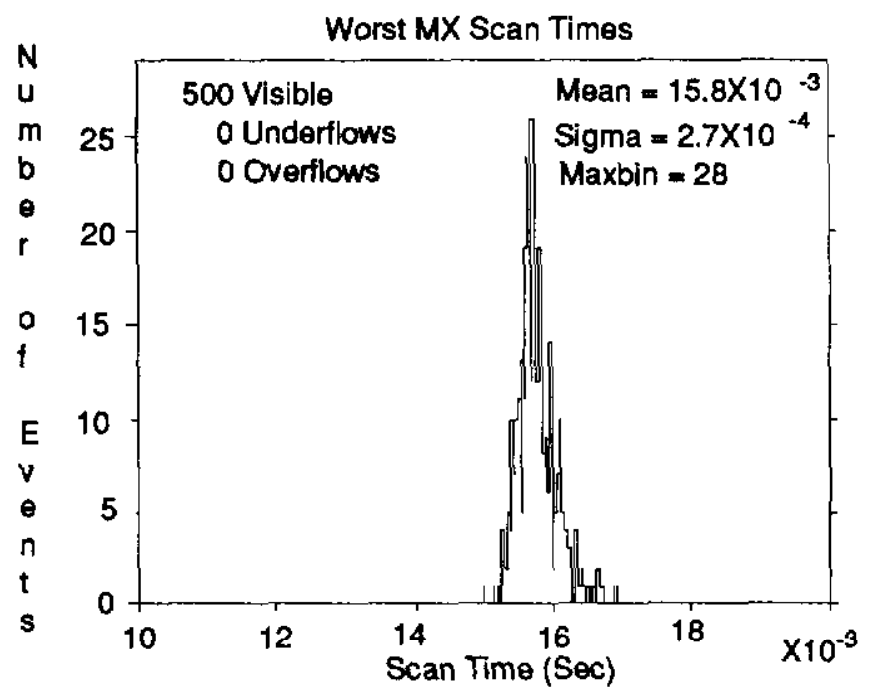

Fig. 4. Worst Case MX Scan Times from a Typical 1989 Physics Run, by Event.

Since runs may be up to ten hours long, pedestal stability is a major concern in this system. Figure 5 shows a histogram of the difference between two pedestal runs for 820 PMADC channels used in the central electromagnetic detector (CEM), taken 5 days apart. The average deviation is 3 ADC counts, where each count corresponds to $38 \mu \mathrm{V}$, or $5.7 \mathrm{MeV}$. Measurements of the reference voltages for all the the EWE modules shows that there was a systematic shift of 2.42 ADC counts throughout the system, so that the actual shift is less than 1 ADC count, out of a full scale range of $65535 \mathrm{ADC}$ counts. This is essentially the same as before the upgrades for the photomultiplier system [7], and indicates that the same high precision measurement capability has been maintained.

The GPA modules for the plug and forward detectors are new in the system. While the GPA modules have all been installed, as of this writing many of the detector components have not yet been commissioned. (The GPA module design has been extensively tested with the detectors in the test beam, where the detectors are undergoing calibration.) However, the electronics has been operated daily since the installation was completed, and two months of experience has been obtained. Figure 6 shows the difference between two pedestal runs for 5035 channels in the plug electromagnetic calorimeter (PEM), taken at the same time as the PMADC pedestal measurements. The average deviation is $6 \mathrm{ADC}$ counts. Correcting for the systematic shift gives a shift of 3.58 ADC counts. The measurement compares favorably with the stability of the PMADC modules. 


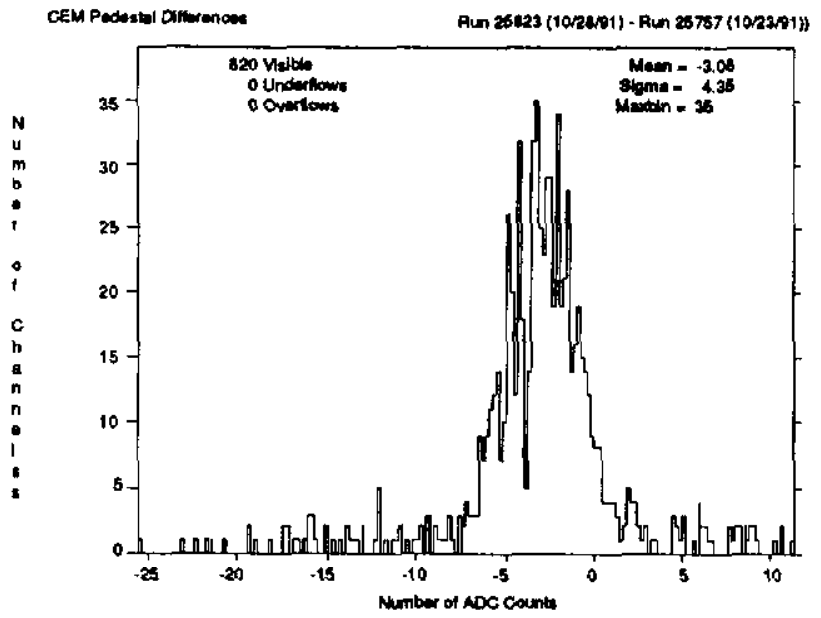

Fig. 5. Deviation in Pedestals for PMADC, 150 Channels, Taken 5 Days Apart.

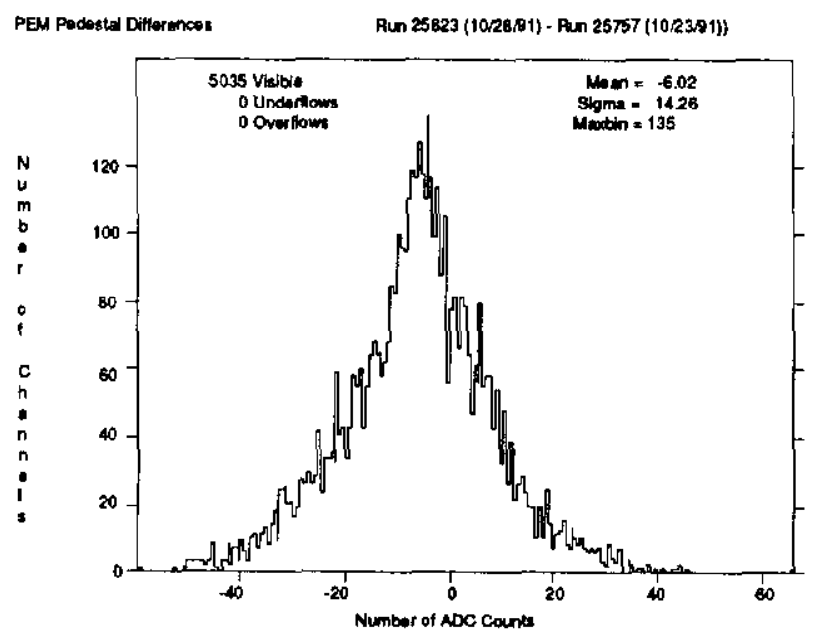

Fig. 6. Deviation in Pedestals for GPA, 5035 Channels, Taken 5 Days Apart.

The trigger system is also not yet commissioned as of this writing, undergoing other modifications and improvements. System-wide tests have not yet been done, although a small number of GPA and PMADC modules have been tested in the system. Based on these tests and the bench tests, the preliminary indications are that the analog signals that are transmitted over the 70 meter fast out cables will settle in time to make a Level 1 trigger decision before the next beam crossing.

\section{SUMMARY}

Upgrades to the data acquisition electronics have been designed and implemented for use with the calorimetry of the CDF experiment to accommodate the higher expected luminosity of the accelerator and to decrease dead time due to the trigger system and the read out of data. Two hundred new digitizers called the EWE III have been produced, and new data acquisition code written, which decrease the dead time due to scanning from $18 \mathrm{mSec}$ to less than $2 \mathrm{mSec}$. Nine hundred new charge-sensitive amplifier modules called the GPA have been produced for the plug and forward calorimeters. The GPA module has an improved amplifier design to obtain better performance to accommodate the new shorter $800 \mathrm{nSec}$ integration time required for the $3.5 \mu \mathrm{Sec}$ collision rate. It also has improved fast out circuitry to obtain better settling of the analog-based trigger signals on the receiving end of the 70 meter trigger cables. The PMADC modules used with the photomultipliers have also had improvements made to the fast out circuitry to improve settling. The trigger system will now be able to make a Level 1 trigger decision before the next beam crossing, which eliminates the dead time due to the Level 1 trigger when the event is rejected.

\section{ACKNOWLEDGEMENTS}

We gratefully acknowledge the fine efforts of the technicians in the Particle Instrumentation Group at Fermilab in the development, production, and installation of the EWE and GPA modules. We also thank the many CDF physicists who helped in commissioning and testing the new system.

\section{REFERENCES}

[1] F. Abe, et al., "The CDF Detector: An Overview," $\mathrm{Nucl}$. Instr. and Meth., vol. A271, pp. 387-403, 1988.

[2] D. Amidei, et al., "A Two Level Fastbus Based Trigger System for CDF," Nucl. Instr. and Meth., vol. A269, pp. 51-62, 1988.

[3] T. M. Shaw, et al., "Performance and System Flexibility of the CDF Hardware Event Builder," these proceedings.

[4] G. Drake, et al., "The RABBIT System," IEEE Trans. Nucl. Sci., vol. 33, pp. 92-97, Feb., 1986.

[5] G. Drake, et al., "CDF Front End Electronics: The RABBIT System," Nucl. Instr. and Meth., vol. A269, pp. 68-81, 1988.

[6] Burr-Brown Integrated Circuits Data Book, vol. 33.

[7] G. Drake, et al., "A Large Dynamic Range Charge Amplifier ADC," IEEE Trans. Nucl. Sci., vol. 3, pp. 893-896, Feb., 1986. 